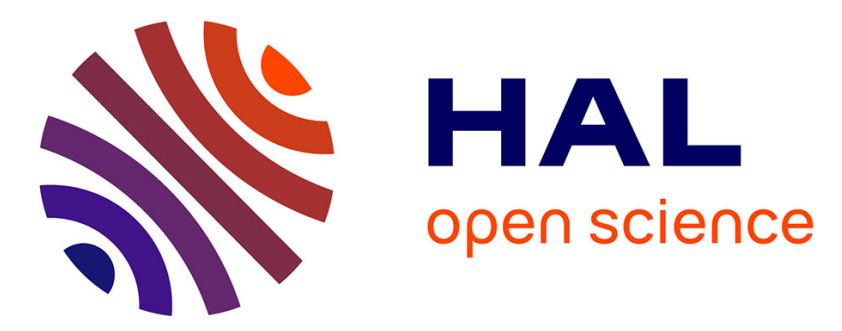

\title{
Acceptabilité des consommateurs guadeloupéens pour l'amélioration de la qualité nutritionnelle des boissons sucrées : une étude expérimentale
}

\author{
Valérie Angeon, Pascale Bazoche, Mathilde Gorza
}

\section{- To cite this version:}

Valérie Angeon, Pascale Bazoche, Mathilde Gorza. Acceptabilité des consommateurs guadeloupéens pour l'amélioration de la qualité nutritionnelle des boissons sucrées : une étude expérimentale. Cahiers de Nutrition et de Diététique, 2022, 57 (1), pp.59-73. 10.1016/j.cnd.2021.11.002 . hal-03528013

\section{HAL Id: hal-03528013 \\ https://hal.inrae.fr/hal-03528013}

Submitted on 17 Jan 2022

HAL is a multi-disciplinary open access archive for the deposit and dissemination of scientific research documents, whether they are published or not. The documents may come from teaching and research institutions in France or abroad, or from public or private research centers.
L'archive ouverte pluridisciplinaire HAL, est destinée au dépôt et à la diffusion de documents scientifiques de niveau recherche, publiés ou non, émanant des établissements d'enseignement et de recherche français ou étrangers, des laboratoires publics ou privés. 
Disponible en ligne sur ScienceDirect www.sciencedirect.com
Elsevier Masson France

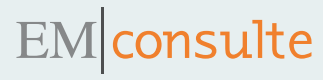

www.em-consulte.com

\title{
Acceptability of Guadeloupean consumers to improve the nutritional quality of sugary drinks: an experimental study
}

\author{
Pascale Bazoche ${ }^{a, *}$, Mathilde Gorza ${ }^{b}$, Valérie Angeon ${ }^{c}$ \\ a INRAE, SMART-LERECO, UMR 1302, 4, allée Adolphe-Bobierre, 35011 Rennes, France \\ b Université Paris-Saclay, INRAE, UR ALISS, 65, boulevard de Brandebourg, 94205 \\ Ivry-sur-Seine, France \\ c INRAE, UR Écodéveloppement, 228, route de l'Aérodrome, CS 40509, Domaine Saint-Paul, \\ Site Agroparc, 84914 Avignon, France
}

Reçu le 27 septembre 2021 ; accepté le 22 novembre 2021

\section{MOTS CLÉS}

Économie

expérimentale ;

Comportement

alimentaire ;

Consentement à

payer ;

Qualité

nutritionnelle ;

Évaluation sensorielle
Résumé L'augmentation des prévalences d'obésité et de maladies chroniques aux Antilles françaises et l'identification d'une transition nutritionnelle avancée nécessitent une compréhension fine des mécanismes d'arbitrages des consommateurs quant à l'offre alimentaire. Nous proposons une étude expérimentale visant à estimer dans quelle mesure les consommateurs guadeloupéens sont prêts à payer pour une amélioration de la qualité nutritionnelle. L'acceptabilité d'une telle amélioration ayant des conséquences (perçues ou supposées) sur les caractéristiques sensorielles, nous proposons une analyse combinant l'évaluation sensorielle à la révélation de consentements à payer pour des boissons sucrées. Les résultats montrent une préférence marquée des consommateurs guadeloupéens pour le sucre dès lors qu'ils peuvent goûter le produit. Le critère sensoriel semble ainsi primer sur l'information nutritionnelle et cela même si l'information nutritionnelle, lorsqu'elle est la seule disponible, n'engendre pas de rejet des produits les moins sucrés.

(c) 2021 Société française de nutrition. Publié par Elsevier Masson SAS. Tous droits réservés.

\footnotetext{
* Auteur correspondant.

Adresse e-mail : pascale.bazoche@inrae.fr (P. Bazoche).
} 


\section{KEYWORDS}

Experimental economics;

Food behaviour; Willingness to pay; Nutritional quality; Sensory evaluation
Summary The increase in the prevalence of obesity and chronic diseases in the French West Indies and the identification of an advanced nutritional transition require a detailed understanding of the mechanisms of consumer trade-offs regarding to the food supply. We propose an experimental study to estimate the extent to which Guadeloupean consumers are willing to pay for an improvement in nutritional quality. As the acceptability of such an improvement has consequences (perceived or assumed) on sensory characteristics, we propose an analysis combining sensory evaluation with the revelation of willingness to pay for sweetened drinks. The results show a marked preference of Guadeloupean consumers for sugar as soon as they can taste the product. The sensory criterion thus seems to take precedence over nutritional information, even though nutritional information, when it is the only one available, does not lead to a rejection of the less sweetened products.

(c) 2021 Société française de nutrition. Published by Elsevier Masson SAS. All rights reserved.

\section{Introduction}

Les Antilles françaises connaissent actuellement un stade avancé de transition nutritionnelle qui se traduit par un profond changement des régimes alimentaires lié à l'évolution de leur contexte économique et social [1] : urbanisation, développement de l'alimentation ultra-transformée, sédentarité, etc. Une transition nutritionnelle se caractérise notamment par une diminution des glucides complexes et une augmentation de la part des protéines et des lipides dans l'alimentation. Cette augmentation peut avoir pour conséquence une augmentation des prévalences d'obésité et des maladies chroniques [2] qui témoigne d'une urgence de santé publique aux Antilles (Livre Bleu Outre-mer, Plan National Nutrition Santé 2019-2023 ${ }^{1}$ ). En effet, en 2010 un tiers des Guadeloupéens étaient concernés par de l'hypertension artérielle [3] et $58 \%$ des adultes étaient en surpoids ou obèses en 2013.

Contrairement à la France hexagonale, il n'y a que peu d'études qui ont été menées dans les territoires d'Outre-mer pour caractériser la transition nutritionnelle [4,5]. Notamment, les changements des comportements alimentaires des populations antillaises sont peu connus et, malgré les efforts des pouvoirs publics pour s'attaquer aux problématiques de santé touchant ces territoires, il reste nécessaire d'identifier les freins et leviers permettant à la population de tendre vers un régime alimentaire plus équilibré.

Parmi les outils classiquement mobilisés pour modifier les comportements alimentaires, figurent les dispositifs de taxation et les outils informationnels. Les uns comme les autres sont encadrés par des directives nationales mais présentent des limites d'efficacité qui s'amplifient dans leur déclinaison outre-mer. En effet, si la taxation nutritionnelle peut être un outil efficace pour induire des changements de comportements alimentaires [6,7], il est cependant important de souligner l'effet régressif d'une telle taxe qui impacterait plus sévèrement les ménages pauvres et pourrait donc de fait induire une diminution de bien-être à court et long terme [8]. Dans le contexte guadeloupéen dans

\footnotetext{
1 https: / / www.vie-publique.fr/rapport/37502-livre-bleu-outre -mer.
}

lequel la pauvreté touche un tiers de la population, une telle politique de taxation ne semble pas appropriée.

De même, les outils informationnels qui peuvent prendre des formes multiples (campagnes d'information, étiquetages nutritionnels, allégations nutritionnelles, etc.) sont couramment utilisés dans l'éducation aux pratiques alimentaires équilibrées. Ils nécessitent néanmoins des efforts cognitifs (lecture, compréhension, appropriation de l'information, réflexion, etc.) de la part des consommateurs. Ces efforts peuvent apparaître aux consommateurs plus ou moins coûteux, ce qui tend potentiellement à limiter l'intérêt des outils informationnels pour l'amélioration des pratiques alimentaires. La littérature démontre en effet le caractère relatif de ces signaux d'information selon le niveau de revenu des consommateurs $[9,10]$ mais aussi de leurs ressources cognitives [11].

Ces principes de réalité amènent à mettre en lumière les inégalités de nutrition au regard des inégalités socioéconomiques [1]. Dès lors, aux Antilles françaises, où il existe de fortes inégalités socioéconomiques (le taux de chômage est deux fois et demie plus élevé en Guadeloupe qu'en Métropole et $41 \%$ de la population Guadeloupéenne est en situation de privation matérielle et sociale [12]), l'adaptation des outils informationnels aux spécificités locales doit être privilégiée pour favoriser leur efficacité.

Par ailleurs, il faut souligner que la qualité nutritionnelle de l'offre alimentaire aux Antilles françaises n'a pas toujours été équivalente à celle de Métropole. En témoigne la loi Lurel, publiée au Journal Officiel en 2013, visant à interdire la différence de teneur en sucres entre la composition des produits manufacturés vendus dans les régions d'outremer et celle des mêmes produits vendus dans l'hexagone. $\mathrm{Si}$ on attend encore les résultats de la Direction générale de la concurrence, de la consommation et de la répression des fraudes (DGCCRF) sur l'évolution de la teneur en sucres des produits vendus en Outre-mer, une récente étude [13] montre que l'offre de produits disponible aux Antilles n'est pas radicalement plus sucrée que celle disponible en France hexagonale. L'alimentation trop riche en sucres relevée dans la population antillaise s'explique essentiellement par la structure des prises alimentaires (composition et rythme). Ainsi, l'écart des apports en sucres entre les Antilles et l'Hexagone peut atteindre 
111 \% (écart donc lié aux quantités consommées plutôt qu'aux teneurs). Sur cette base, une appréhension des connaissances et des croyances des individus en matière alimentaire est susceptible de favoriser l'élaboration de mesures pertinentes pour infléchir les comportements de consommation.

Dans cet article, nous nous interrogeons sur les déterminants individuels des choix d'achat et de consommation alimentaires en Guadeloupe. Compte tenu de la place qu'occupent les boissons sucrées dans l'alimentation de la population guadeloupéenne $[1,13]$ et de leurs impacts délétères sur la santé, nous nous intéressons à l'acceptabilité des boissons à teneur en sucres moins élevée. L'acceptabilité d'une telle amélioration ayant des conséquences (perçues ou supposées) sur les caractéristiques sensorielles, nous proposons une analyse combinant l'évaluation sensorielle à la révélation de consentements à payer.

Afin de déterminer dans quelle mesure l'argument « nutritionnel » peut être un levier sur la demande, nous complétons nos données expérimentales par un questionnaire permettant d'identifier les capacités des consommateurs à comprendre un étiquetage nutritionnel et permettant de mieux appréhender les croyances et connaissances de ces consommateurs sur les recommandations nutritionnelles fournies par le Plan National Nutrition Santé (PNNS).

\section{Matériels et méthodes}

La méthodologie adoptée pour cette étude sur l'acceptabilité des consommateurs guadeloupéens pour l'amélioration de la qualité nutritionnelle des produits repose sur la combinaison de plusieurs méthodes : l'économie expérimentale avec révélation de consentements à payer, l'analyse sensorielle et l'étude des croyances, connaissances et habitudes alimentaires.

Les participants à cette étude ont chacun assisté à une session d'économie expérimentale. Une expérience économique consiste à reproduire une situation économique simplifiée dans laquelle l'expérimentateur contrôle l'ensemble des variables intervenant dans cette situation. La situation économique est ici l'achat de produits alimentaires ; les variables contrôlées par l'expérimentateur sont les informations disponibles sur les produits lors de l'acte d'achat. Ainsi, cette approche permet de comprendre comment chaque individu détermine la valeur globale d'un produit à partir de la valeur de ses différentes caractéristiques [14] non pas en se basant sur des données déclaratives mais en se basant sur des comportements réels d'achat.

De plus, il est particulièrement pertinent de coupler une expérimentation d'économie expérimentale avec une analyse sensorielle car les caractéristiques sensorielles d'un produit impactent incontestablement la décision finale de consentement à payer et donc le choix d'achat [15-17]. C'est pourquoi une attention particulière a été portée sur l'association des mesures sensorielles et économiques dans notre étude.

Enfin, des éléments d'appréciation des connaissances et des croyances des consommateurs quant aux recommandations nutritionnelles sont intégrés à l'analyse, étant démontré qu'ils jouent un rôle dans les décisions d'achat et de consommation [18].

\section{Recrutement des consommateurs}

Les expérimentations ont été conduites en mars 2021 dans une salle d'analyse sensorielle permettant des évaluations individuelles sans interactions entre les participants. Des consommateurs ont été recrutés par le biais d'opérations de recrutement ciblées compte tenu des caractéristiques de l'échantillon recherché. Les critères de recrutement portaient sur la consommation et l'achat de produits du même type que ceux faisant l'objet de l'étude, et visaient à s'assurer que les participants souhaitaient se prêter à une expérience économique, avec un potentiel achat de produit. De même, seuls des participants majeurs, vivant en Guadeloupe et ne suivant pas de régime alimentaire impactant leur consommation de sucres pouvaient prendre part à l'expérience. Enfin, le recrutement des participants a volontairement été orienté vers les jeunes (entre 18 et 30 ans) qui représentent la classe de la population la plus touchée par la transition nutritionnelle avec une consommation accrue de produits gras, sucrés, salés et transformés. De plus, la consommation de boissons sucrées étant décroissante avec l'âge [1], il paraît pertinent de cibler prioritairement la tranche de population la plus consommatrice du produit étudié. Au total, 179 individus âgés de 18 à 70 ans ont participé aux sessions expérimentales. Pour l'analyse des résultats, les données de 159 participants, dont les caractéristiques sociodémographiques sont présentées dans le Tableau 1, ont été retenues parmi les $179^{2}$.

\section{Sélection des produits}

L'objectif étant de déterminer dans quelle mesure les consommateurs guadeloupéens sont prêt à accepter une amélioration de la qualité nutritionnelle, nous nous sommes intéressé à un secteur particulièrement présent dans les Antilles Françaises, celui des boissons sucrées qui constituent un des principaux vecteurs d'exposition au sucre dans les Antilles $[1,13]$.

Le choix du produit à évaluer s'est porté sur une boisson sucrée, précisément le nectar de goyave, qui est une boisson aux fruits particulièrement consommée en Guadeloupe. Au total, quatre nectars de goyaves ont été utilisés pour l'étude : trois nectars de goyave dont les formulations ont été spécialement conçues pour l'étude et un produit du commerce largement consommé en Guadeloupe (taux de glucides totaux : $9 \mathrm{~g} / 100 \mathrm{~mL}$ ). Les trois nectars spécialement conçus pour notre étude ont tous été produits à partir des mêmes matières premières et selon le même processus de production, la teneur en sucre étant la seule donnée variant dans la composition. Le premier nectar (appelé High)

\footnotetext{
2 Un vingtaine d'observations n'ont pas été retenues du fait de données manquantes ou de réponses incohérentes. En effet, durant les sessions expérimentales, une attention particulière a été portée sur l'observation des participants pendant le premier temps de l'étude, afin d'identifier si l'exercice avait été compris et correctement réalisé. Cette observation a permis de discriminer les participants et de les inclure ou non dans la base de données.
} 
Tableau 1 Caractéristiques sociodémographiques des sujets ayant participés à l'expérience $(n=159)$ et données des issues du recensement Guadeloupe 2018.

\begin{tabular}{|c|c|c|}
\hline Caractéristiques sociodémographiques & $\begin{array}{l}\text { Fréquence (\%) } \\
\text { Dans } \\
\text { l'échantillon } \\
\text { mobilisé }\end{array}$ & $\begin{array}{l}\text { Données INSEE } \\
\text { recensement } \\
\text { Guadeloupe } \\
2018\end{array}$ \\
\hline \multicolumn{3}{|l|}{ Sexe } \\
\hline Homme & 37,74 & 45,94 \\
\hline Femme & 62,26 & 54,05 \\
\hline \multicolumn{3}{|l|}{ Catégories d'âge } \\
\hline Moins de 30 ans & 40,25 & 34,86 \\
\hline Entre 30 et 39 ans & 19,50 & 9,88 \\
\hline Entre 40 et 49 ans & 17,61 & 13,96 \\
\hline Entre 50 et 59 ans & 16,35 & 15,80 \\
\hline 60 ans et plus & 6,29 & 25,51 \\
\hline \multicolumn{3}{|l|}{ Niveau de diplôme } \\
\hline Sans diplôme & 1,26 & 35,4 \\
\hline Brevet des collèges (BEPC) & 2,52 & 5,9 \\
\hline BEP, CAP ou équivalent & 11,32 & 18,2 \\
\hline Baccalauréat (Bac) & 35,22 & 17,8 \\
\hline BTS, DEUG, licence ou équivalent (Bac +2 à Bac +3) & 30,19 & 9,5 \\
\hline Ingénieur ou Master ou équivalent (Bac +4 à Bac +5) & 18,24 & 7,9 \\
\hline Doctorat ou équivalent (Bac +6 et au-delà) & 1,26 & 5,3 \\
\hline \multicolumn{3}{|l|}{ Situation professionnelle } \\
\hline Étudiant & 23,27 & 11,5 \\
\hline À la recherche d'emploi & 16,35 & 20,3 \\
\hline En emploi & 53,46 & 50,3 \\
\hline Autres inactifs & 6,92 & 13,9 \\
\hline \multicolumn{3}{|l|}{ Catégories socioprofessionnelles (actifs uniquement $n=114$ ) } \\
\hline Agriculteurs exploitants & 2,63 & 1,91 \\
\hline Artisans, commerçants et chefs d'entreprise & 10,53 & 11,00 \\
\hline Cadres et professions intellectuelles supérieures (avocat, médecin, architecte, etc.) & 17,54 & 11,58 \\
\hline Professions intermédiaires (infirmière, instituteur, secrétaire de direction, etc.) & 28,95 & 25,10 \\
\hline Employés (vendeur, caissier, assistante maternelle, facteur, etc.) & 35,09 & 33,82 \\
\hline Ouvriers (jardinier, maçon, magasinier, etc.) & 5,26 & 16,52 \\
\hline \multicolumn{3}{|l|}{ Revenus du foyer } \\
\hline Moins de 450 euros & 5,66 & nd \\
\hline Entre 451 et 1200 euros & 27,04 & nd \\
\hline Entre 1201 et 2300 euros & 33,96 & nd \\
\hline Entre 2301 et 3800 euros & 21,38 & nd \\
\hline Entre 3801 et 4500 euros & 10,06 & nd \\
\hline Plus de 4500 euros & 7,55 & nd \\
\hline \multicolumn{3}{|l|}{ Résidence en Guadeloupe } \\
\hline Depuis moins de 3 ans & 6,29 & nd \\
\hline Entre 3 et 10 ans & 5,03 & nd \\
\hline Depuis plus de 10 ans & 22,01 & nd \\
\hline Depuis toujours & 66,67 & nd \\
\hline
\end{tabular}

nd : non disponible.

contient $10 \mathrm{~g}$ de sucre ajouté pour $100 \mathrm{~mL}$, cette teneur en sucre a été choisie afin de correspondre à ce qui est présent en moyenne sur le marché guadeloupéen. Le second nectar contient $7 \mathrm{~g}$ de sucre ajouté pour $100 \mathrm{~mL}$, nous avons choisi cette teneur car elle permettrait de pouvoir ajouter une allégation «allégé en sucre » sur l'étiquette. Enfin, le troisième nectar contient $6 \mathrm{~g}$ de sucre ajouté pour $100 \mathrm{~mL}$ afin de déterminer si une différence marginale de la teneur en sucre est perçue par les consommateurs.

\section{Protocole expérimental}

\section{Révélation des préférences et mécanisme incitatif}

La mesure de l'acceptabilité est réalisée par l'évaluation du consentement à payer pour les 3 produits spécifiquement formulés pour notre étude. Le consentement à payer est le montant maximum qu'un consommateur est disposé à payer 
pour s'approprier un bien ou un service après avoir évalué les conséquences aussi bien positives que négatives en termes de bien-être qu'engendre son acte d'achat. L'évaluation de ces produits est réalisée en laboratoire d'analyse sensorielle par un mécanisme de révélation des préférences incitant à révéler les prix maximums que les consommateurs sont prêts à payer pour les produits qui leur sont proposés.

Afin de s'assurer que les prix maximums des consommateurs soient proches de la réalité, nous utilisons le mécanisme d'incitation BDM [19] qui consiste à mettre en vente les produits en fin de session à un prix de vente tiré au sort. Ainsi, des produits sont proposés aux participants et ces derniers indiquent le prix maximum qu'ils sont prêts à payer pour chaque produit proposé (ce prix peut-être nul s'ils ne veulent pas acheter le produit). Le prix de vente du produit sera tiré au sort en fin de séance dans une urne contenant une distribution de prix dont la moyenne est égale au prix moyen relevé en magasin pour le type de produit proposé.

Les participants dont le consentement à payer est supérieur ou égal au prix de vente (tiré au sort) achètent réellement le produit au prix de vente tiré au sort. Ils doivent être satisfaits de cette situation puisqu'ils acquièrent un produit à un prix inférieur ou égal au prix maximum qu'ils étaient prêts à payer. Inversement, les participants ayant indiqué un consentement à payer inférieur au prix de vente tiré au sort ne peuvent pas acheter le produit. Ils doivent également être satisfaits de cette situation (et cela même si le prix tiré au sort est très faible) puisqu'au final, ils n'ont pas à acheter un produit dont le prix de vente est supérieur à leur prix d'achat maximum. Ainsi, quel que soit le prix de vente, les participants doivent être satisfaits de l'issue de la session qu'il y ait achat du produit ou non.

Afin de nous assurer de la bonne compréhension du dispositif d'incitation, le mécanisme de vente a fait l'objet d'explications détaillées et d'une phase d'entraînement sur un produit fictif. Il s'agit d'un mécanisme incitatif dans lequel les participants sont mis en situation d'achat réel permettant d'impliquer pleinement le consommateur dans l'expression de son prix maximum d'achat et donc d'annihiler le biais déclaratif. De plus, cette méthode est particulièrement intéressante car facile à mettre en œuvre et à intégrer par les participants [15].

\section{Présentation du déroulement des sessions}

Les sessions se sont déroulées en deux temps : premièrement l'expérimentation avec consentement à payer et évaluation sensorielle selon différents types et degrés d'informations, et deuxièmement le questionnaire permettant de mesurer les connaissances, croyances et habitudes des consommateurs. Le protocole expérimental est décrit par la Fig. 1.

\section{Évaluation des nectars : révélation des préférences}

Lors de l'évaluation des nectars, les participants ont révélé leurs préférences dans différentes situations informationnelles. Chaque situation était différenciée par le niveau d'information mis à disposition des participants : dégustation, lecture d'informations nutritionnelles puis les deux simultanément. Le contrôle de l'information donnée permet donc de comprendre le poids de l'information dans l'expression des consentements à payer des consommateurs. Ainsi les participants ont révélé leurs consentements à payer pour 3 nectars de goyave dans 3 situations informationnelles différentes. Dans chaque situation informationnelle, les 3 nectars de goyave ont été présentés dans un ordre aléatoire. Lors de la première situation informationnelle (nommée Blind, situation 1), les consommateurs dégustaient les trois produits en ne détenant aucune information particulière autre que les caractéristiques sensorielles qu'ils percevaient. Dans une seconde situation (nommée Label, situation 2), les consommateurs ont révélé leurs consentements à payer en ne disposant que des étiquettes des produits sur lesquelles étaient mentionnées : la liste des ingrédients et la table des apports nutritionnels. Dans une troisième situation (nommée Full, situation 3), les consommateurs ont à nouveau révélé leurs consentements à payer pour les trois produits mais en disposant de la dégustation du produit et de l'information fournie par l'étiquette.

L'évaluation des nectars s'est terminée par une phase d'évaluation hédonique uniquement (situation 4). Dans cette étape un produit disponible en grande distribution a été ajouté afin de pouvoir caractériser les différences perçues entre les nectars spécifiquement produits pour l'étude et ceux disponibles en grande distribution. Pour chaque produit dégusté, le consommateur a été amené à évaluer son appréciation globale du produit selon une échelle hédonique en 5 points (Vraiment pas bon - Pas très bon - Moyen - Assez bon - Très bon) ainsi qu'à évaluer les nectars suivant différents descripteurs sensoriels (sucre, acidité, texture, goût fruité) selon une échelle JAR en 5 points (Pas assez - Manque un peu - Juste bien - Un peu trop - Trop). Cette situation d'évaluation a été insérée en dernière étape afin d'appréhender le lien entre les évaluations économiques (par le biais de la révélation des consentements à payer) et les évaluations hédoniques d'attributs sensoriels spécifiques.

Les évaluations successives répétées pouvant créer un effet de lassitude des participants, les évaluations économiques ont été réalisées en premier afin d'assurer un niveau de concentration élevé des participants lors de ces évaluations. En effet, le mécanisme de prix de vente aléatoire n'étant pas classique pour les participants et représentant une tâche cognitive plus complexe, il a semblé plus approprié de privilégier cet ordonnancement.

\section{Questionnaire : mesure des connaissances et croyances}

Dans un second temps, l'expérience a été complétée par un questionnaire abordant les thèmes suivants :

- connaissances en nutrition : questions autour des recommandations du Programme National Nutrition Santé ${ }^{3}$, définition de certaines appellations et allégations nutritionnelles, lecture d'informations nutritionnelles. Il s'agit

\footnotetext{
${ }^{3}$ Le Programme National Nutrition Santé (PNNS) a pour objectif général l'amélioration de l'état de santé de l'ensemble de la population en agissant sur l'un de ses déterminants majeurs : la nutrition.
} 


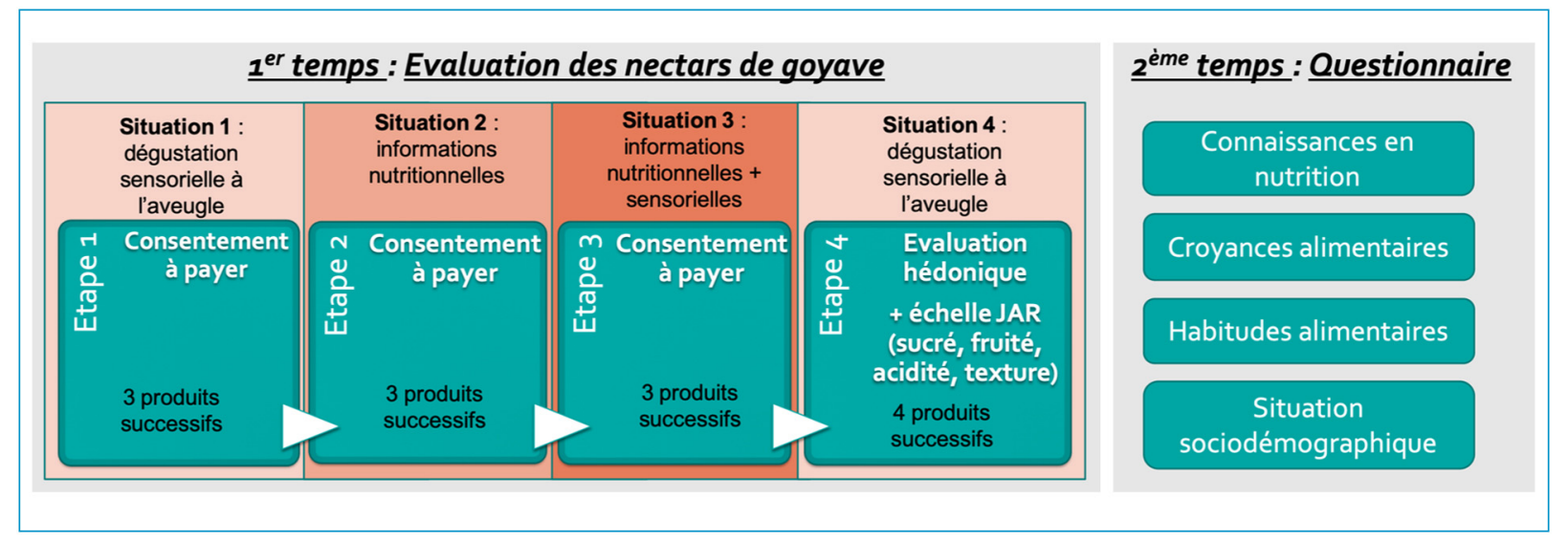

Figure 1. Protocole expérimental décrivant le déroulement d'une session.

de savoirs objectifs pouvant être évalués par des critères scientifiques stabilisés ;

- croyances alimentaires : identification des croyances en termes de consommation de boissons sucrées et impact sur la santé, consommation locale, activité physique. Les croyances renvoient à des représentations, des visions du monde partagées ou non. Elles sont éminemment subjectives ;

- habitudes alimentaires : régime alimentaire, types, fréquence, quantité et mode de consommation de boissons fruitées. On entend par là les choix récurrents observés par les individus quant aux aliments qu'ils consomment ;

- situation sociodémographique : sexe, date de naissance, nombre d'années de résidence en Guadeloupe, situation maritale, situation professionnelle actuelle, diplôme le plus élevé obtenu, nombre de personnes vivant dans le foyer, nombre d'enfants de moins de 18 ans, revenu mensuel net du foyer. Il s'agit des critères classiques permettant entre autres de décrire les individus selon le genre et la position socioéconomique.

\section{Résultats}

L'analyse des résultats a pour objectif de voir quel(s) critère(s) influence(nt) le prix maximum d'achat des consommateurs (taux de sucres, informations nutritionnelles, autres caractéristiques sensorielles, etc.) et d'identifier leurs préférences et habitudes alimentaires.

Les résultats se structurent en quatre parties. La première partie présente les résultats des consentements à payer recueillis pour chacun des nectars et dans les différentes conditions d'évaluation durant la session d'économie expérimentale (étapes 1 à 3 ). La seconde partie concerne les résultats de l'évaluation sensorielle des différents nectars de goyave (étape 4). En troisième partie sont abordés les résultats du questionnaire complémentaire soumis aux participants. Enfin, la quatrième partie met en relation les résultats des parties précédentes pour identifier les variables déterminantes du consentement à payer et leur poids.

\section{Analyse descriptive des consentements à payer}

Cette partie présente les résultats des consentements à payer exprimés par les participants pour chaque situation et chaque nectar évalué. Le Tableau 2 synthétise les statistiques descriptives appliquées sur les consentements à payer.

On constate qu'en moyenne, les consentements à payer sont plus élevés en situation d'information complète (étiquette avec dégustation) qu'en information partielle. Cependant, à la seule observation des moyennes, on constate que cette valorisation accrue en information complète n'est pas systématique et varie selon la teneur en sucre du nectar évalué. Ainsi, les consentements à payer (CAP) ont été soumis aux tests statistiques non paramétriques de Mann-Whitney permettant de vérifier les différences significatives entre les moyennes des CAP obtenues pour chaque nectar intra situations. La Fig. 2 représente les CAP moyens exprimés pour chacun des nectars et selon les trois situations, ainsi que la comparaison par test de Mann-Whitney. En situation 1, le nectar le plus sucré (High) est valorisé de manière significative par rapport aux nectars intermédiaire (Med) et moins sucré (Low). En situation 2, le nectar dont le CAP moyen est le plus élevé est le nectar intermédiaire, significativement supérieur au nectar le plus sucré avec un taux de significativité de $10 \%$. Enfin, en situation 3 , le nectar le plus sucré est de nouveau le nectar le plus valorisé avec un CAP moyen largement supérieur aux deux autres nectars (seuil de significativité de $1 \%$ ). Ainsi on constate qu'avec la seule caractéristique sensorielle, le nectar le plus sucré est de loin préféré, cet écart en faveur du produit le plus sucré persiste dès lors que la révélation du consentement à payer inclue la dimension sensorielle en complément de la dimension informationnelle.

La Fig. 3 illustre les dynamiques de choix des nectars par les participants de la première à la troisième situation. Dans ce graphique, le nectar préféré par le consommateur est défini par le CAP le plus élevé qui a été indiqué entre les trois nectars par le participant pour chaque situation informationnelle. Dans le cas où deux nectars ont été évalués au même prix maximum, nous avons émis l'hypothèse que 
Tableau 2 Moyennes, écart-types, min et max de consentements à payer par situation et par teneur en sucre. $n=159$.

\begin{tabular}{lllll}
\hline Consentements à payer (en euros) & Moyenne & Écart-type & Minimum & Maximum \\
\hline Situation 1 - Blind - tous nectars & 1,38 & 0,87 & 0 & 5,00 \\
Nectar « High » & 1,63 & 0,90 & 0 & 5,00 \\
Nectar « Med» & 1,30 & 0,83 & 0 & 4,00 \\
Nectar « Low » & 1,19 & 0,81 & 0 & 4,00 \\
Situation 2 - Label - tous nectars & 1,43 & 0,77 & 0 & 4,50 \\
Nectar « High » & 1,35 & 0,76 & 0 & 4,00 \\
Nectar « Med» & 1,48 & 0,75 & 0 & 4,00 \\
Nectar « Low » & 1,44 & 0,80 & 0 & 5,00 \\
Situation 3 - Full - tous nectars & 1,48 & 0,83 & 0 & 4,00 \\
Nectar « High » & 1,69 & 0,86 & 0 & 4,50 \\
Nectar « Med» & 1,39 & 0,82 & 0 & 5,00 \\
Nectar « Low » & 1,35 & 0,76 & 0 & \\
\hline
\end{tabular}

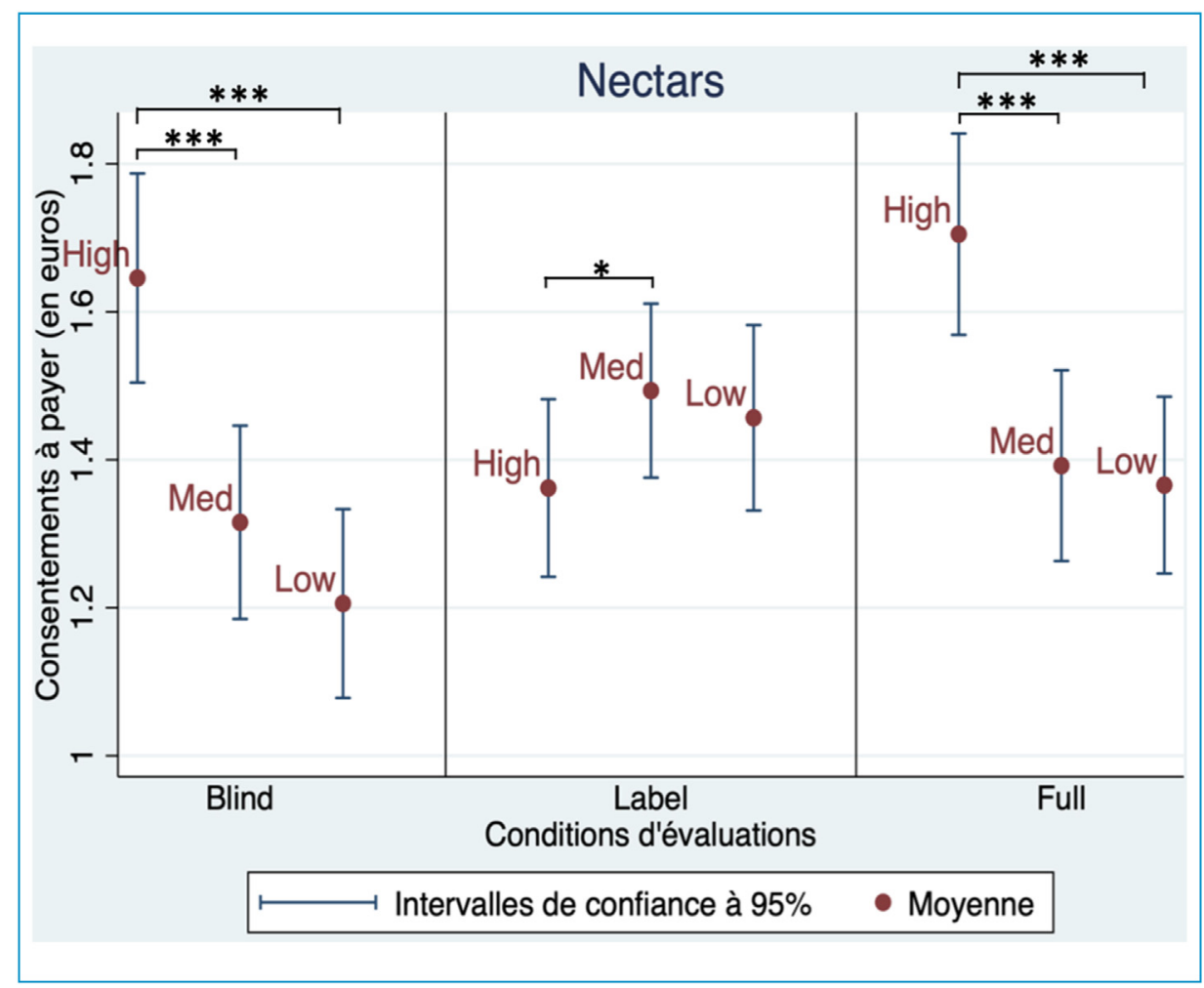

Figure 2. Moyennes des consentements à payer par teneur en sucre et situation informationnelle. $n=159$. ${ }^{* * *}$ différence significative par le test de Mann-Whitney au seuil de $1 \%$, * au seuil de $10 \%$.

le nectar préféré était le plus sucré ${ }^{4}$. Le graphique se lit en partant des choix finaux représentés en situation 3 pour remonter vers la situation 2 et 1 .

Les bandes rouges représentent les consommateurs ayant préféré le nectar le plus sucré en situation finale (étiquette et dégustation), les bandes bleues les participants ayant préféré le nectar au taux de sucre intermédiaire en situation 3, les bandes vertes représentent ceux ayant préféré le nectar le moins sucré en situation 3 . Au total, $66 \%$ des participants

\footnotetext{
${ }^{4}$ Une deuxième hypothèse qui considère que le nectar préféré serait le moins sucré a également été testée et les dynamiques de choix suivent la même tendance.
}

ont préféré le nectar le plus sucré en situation de dégustation à l'aveugle (Blind) et $68 \%$ en situation d'information complète (Full). Il apparaît donc que le nectar le plus sucré est fortement valorisé en situation 1 et 3 , ce qui suggère que les critères sensoriels priment sur l'aspect nutritionnel dans le choix des consommateurs. Au total, presque la moitié de l'échantillon ( $46 \%$ ) a préféré le nectar le plus sucré à l'aveugle et en situation complète.

Cette représentation graphique montre que si les préférences pour le nectar le plus sucré sont marquées lorsque les participants prennent en compte la dimension sensorielle, par contre les préférences entre les trois niveaux de sucre proposés sont réparties équitablement lorsque 


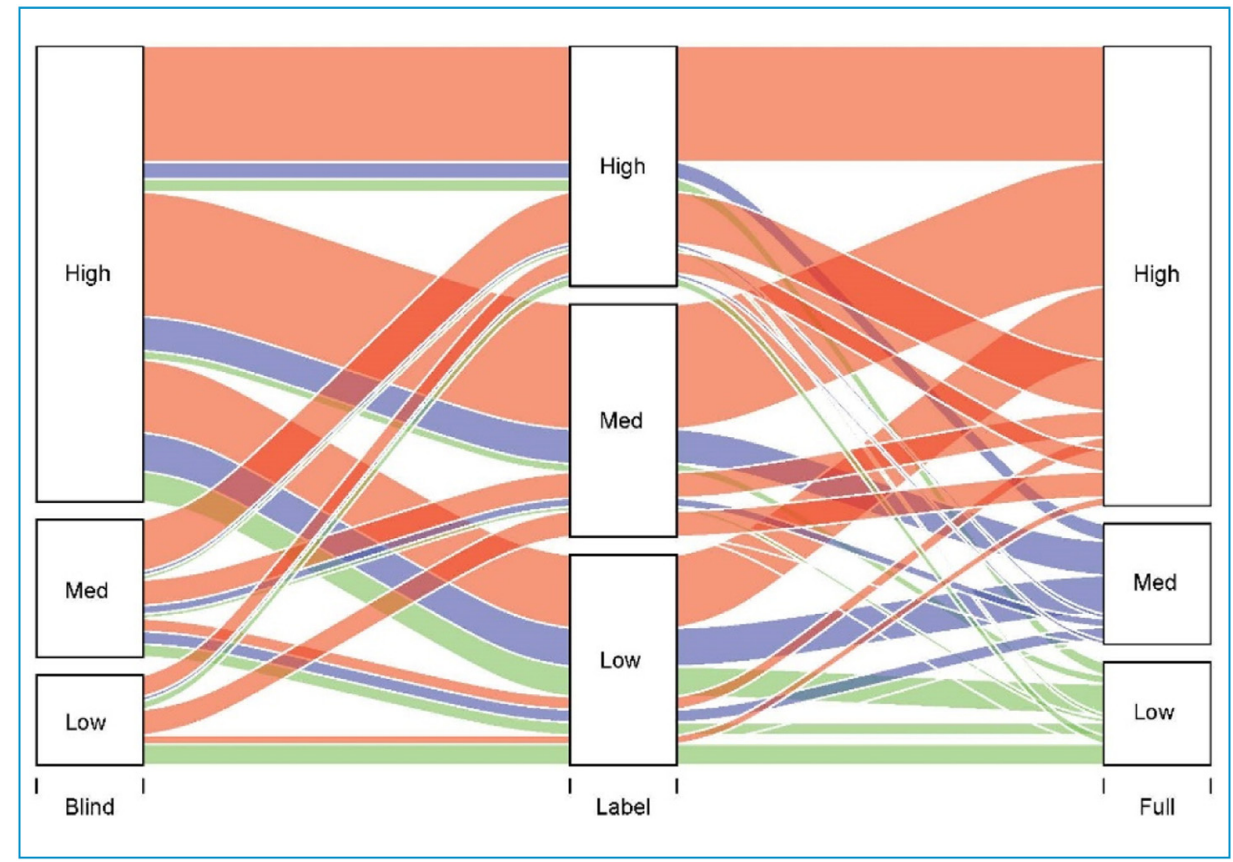

Figure 3. Graphique alluvial représentant les dynamiques de choix des nectars par teneur en sucre. $n=159$.

seule l'information sur les apports nutritionnels est donnée (Label).

\section{Analyse descriptive de l'évaluation sensorielle}

La dernière situation d'évaluation des nectars s'est effectuée à l'aveugle. Elle consistait à noter les quatre nectars selon leur appréciation globale (note hédonique) et quatre descripteurs sensoriels (sucré, acide, caractère liquide et caractère fruité). Un quatrième nectar a été évalué par les participants, il s'agit d'un produit industriel, communément consommé en Guadeloupe. Ce produit supplémentaire a été ajouté pour cette dernière situation afin d'observer et comparer les notations de ce nectar par rapport aux trois autres spécialement produits par un artisan local.

La Fig. 4 présente les moyennes et intervalles de confiances de ces descripteurs, ainsi que les résultats du test de Mann-Whitney. L'appréciation globale hédonique est obtenue par une échelle hédonique en 5 points de «Vraiment pas bon » à « Très bon ». Les évaluations des autres descripteurs sensoriels (caractère sucré, acide, liquide et fruité) sont obtenues par une échelle JAR en 5 points ( 1 = Pas assez ; 2 = Manque un peu ; 3 = Juste bien ; 4 = Un peu trop ; 5 = Trop).

Les résultats montrent que lors de l'évaluation hédonique globale des produits, le nectar le plus sucré est significativement plus apprécié que les autres. Le nectar du commerce est faiblement valorisé, ce qui va à l'encontre de ce qui était attendu, ce dernier ayant une teneur en sucre similaire au plus sucré des nectars produits pour l'expérience.

Il ressort de la Fig. 4 une appréciation positive du sucre dans le nectar industriel et le nectar le plus sucré, qui sont tous les deux notés autour de 3, c'est-à-dire proche de «Juste bien ». Il y a une différence très significative de perception du caractère sucré entre le nectar le plus sucré et les deux autres moins sucrés. Il n'y a pas de différence significative entre le nectar intermédiaire et le nectar le moins sucré, ce qui peut être expliqué par la formulation des produits : l'écart du taux de sucre entre le nectar «Med » et «Low » (respectivement $7 \mathrm{~g}$ et $6 \mathrm{~g}$ pour $100 \mathrm{~mL}$ ) est bien inférieur à celui entre le nectar « High » et «Med» (respectivement $10 \mathrm{~g}$ et $7 \mathrm{~g}$ pour $100 \mathrm{~mL}$ ).

Concernant l'évaluation du caractère acide des quatre nectars, les notes moyennes des nectars sont proches (entre 2,7 et 3,4 ). On peut noter que le nectar le plus sucré est le mieux noté (très proche de 3 ), ce qui correspond à une évaluation « Juste bien ». Le nectar industriel est perçu comme moins acide que les autres, ce qui pourrait être dû à la formulation différente du produit par rapport aux trois autres spécialement fabriqués pour l'expérimentation.

Lorsque l'on s'intéresse à l'évaluation du caractère liquide des quatre nectars, la texture la plus appréciée par les consommateurs est celle du nectar industriel, ce qui peut aussi être expliqué par la formulation différente de ce produit par rapport aux autres. Le nectar perçu comme le plus liquide est le nectar intermédiaire, et non pas le moins sucré, ce qui va à l'encontre du résultat attendu.

Enfin, pour l'évaluation du caractère fruité des quatre nectars, on remarque des résultats très similaires à ceux obtenus pour l'évaluation du caractère sucré, ce qui laisse penser que les participants assimilent le goût fruité d'un produit à son goût sucré.

\section{Analyse descriptive des connaissances et croyances}

Les résultats du questionnaire sont présentés selon les différents thèmes abordés : connaissances en nutrition, croyances alimentaires et habitudes alimentaires. 


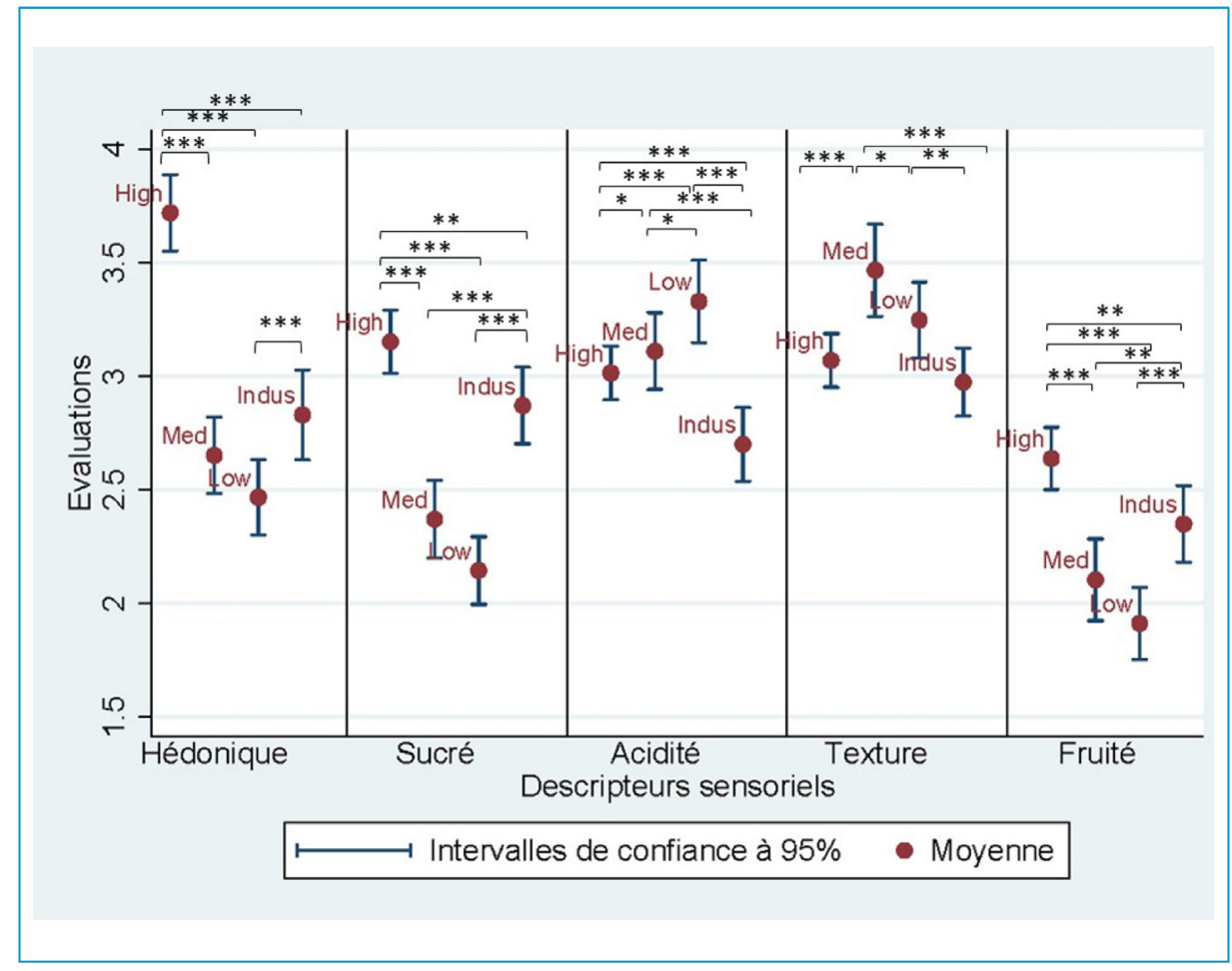

Figure 4. Moyennes et intervalles de confiances des évaluations par descripteur sensoriel. $n=159 .{ }^{* * *}$ différence significative par le test de Mann-Whitney au seuil de $1 \%$, ${ }^{* *}$ différence significative par le test de Mann-Whitney au seuil de $5 \%$, * différence significative par le test de Mann-Whitney au seuil de $10 \%$.

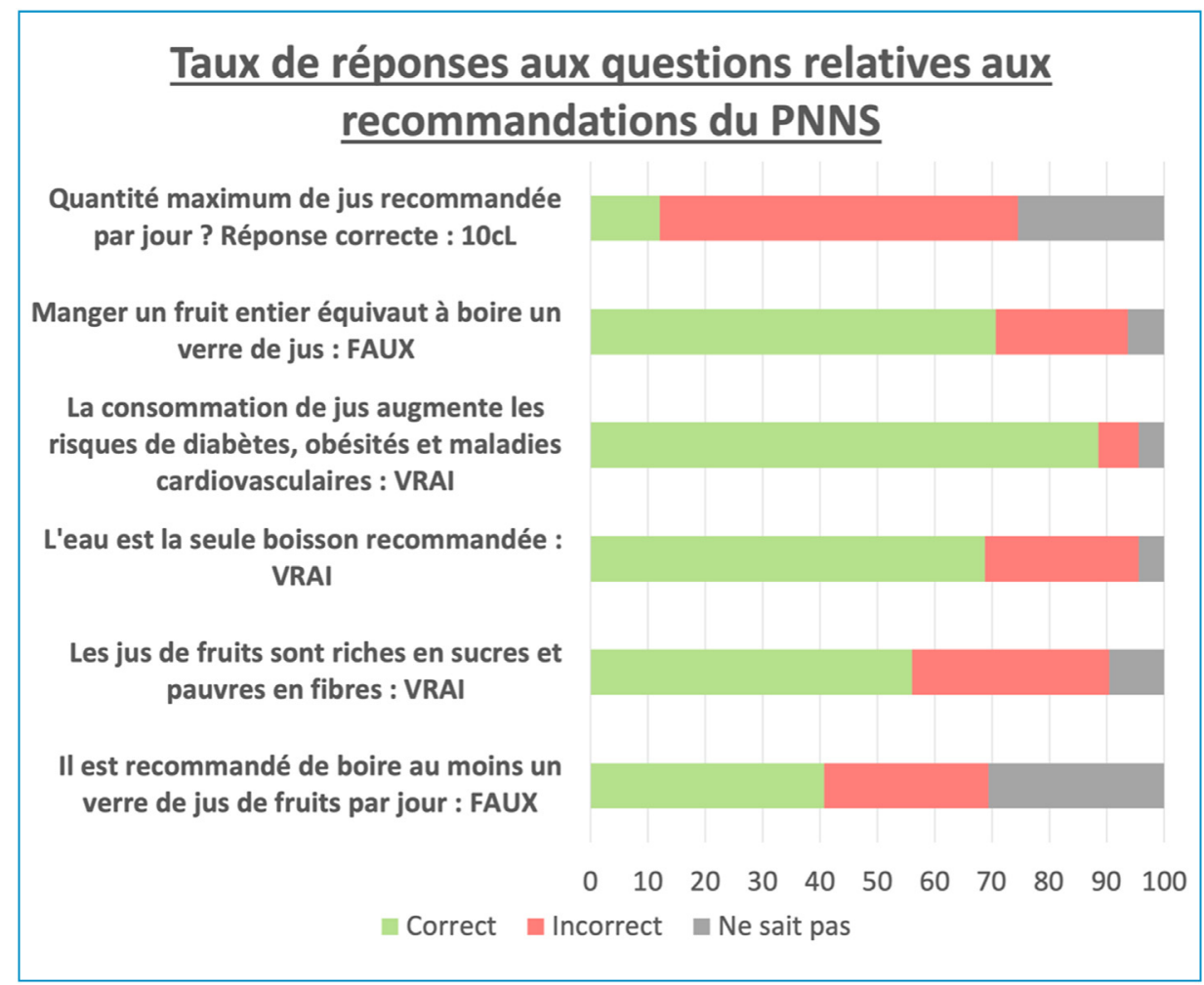

Figure 5. Taux de réponses (correctes/incorrectes/non connues) des participants concernant les questions relatives aux recommandations du PNNS. $n=159$. 


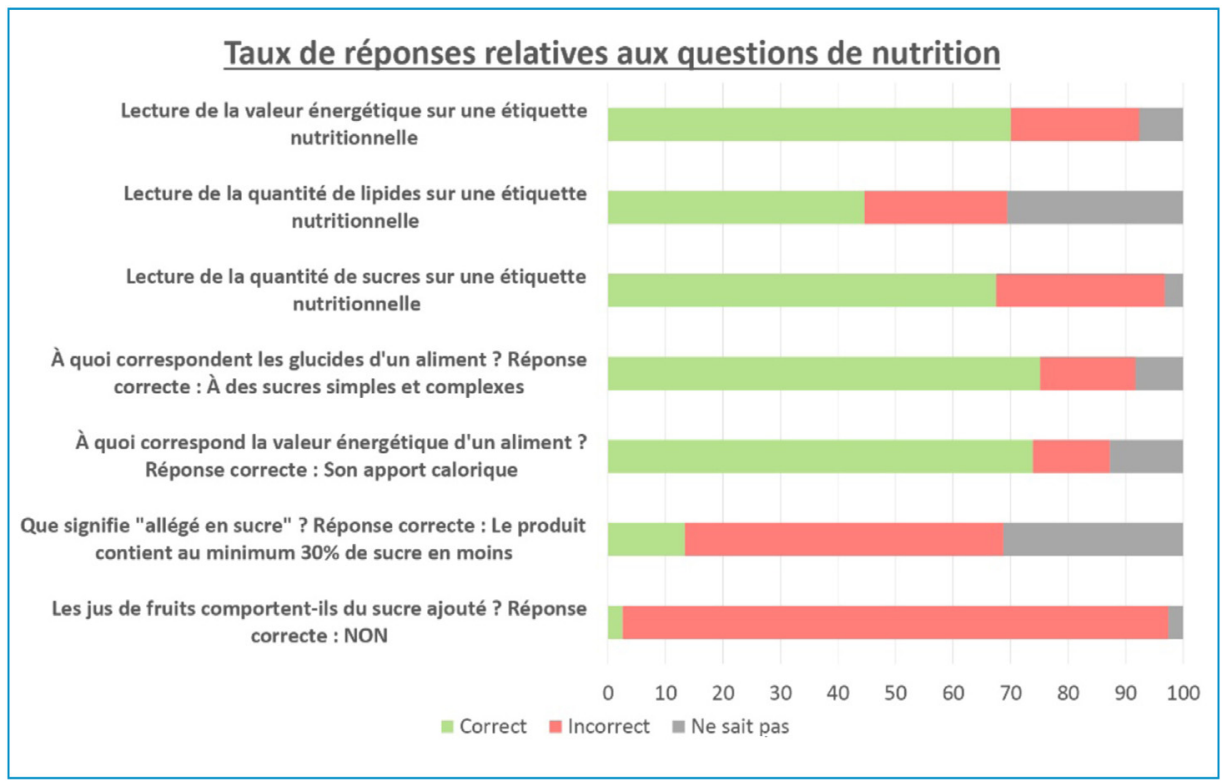

Figure 6. Taux de réponses (correctes/incorrectes/non connues) des participants concernant les questions relatives à la lecture d'une étiquette nutritionnelle et la connaissance de termes de nutrition. $n=159$.

\section{Des recommandations peu connues}

Lancé en 2001, le PNNS est un plan de santé publique visant à améliorer l'état de santé de la population en agissant sur la nutrition qui est un facteur majeur de protection de la santé. Ces recommandations sont actualisées tous les 4 ans selon des objectifs nutritionnels de santé publique. Le PNNS actuel (2019-2023) est le quatrième à être mis en œuvre. Dans l'étude menée, différentes questions portaient sur la connaissance des recommandations incluses dans le PNNS actuel. La Fig. 5 présente pour chaque question la répartition des réponses énoncées par les participants.

Concernant la quantité maximale de consommation de boissons à base de fruits recommandée par jour, seulement $12 \%$ des participants ont répondu $10 \mathrm{CL}$ par jour, ce qui correspond à la quantité indiquée dans le PNNS. De plus, le PNNS recommande de ne pas dépasser la consommation d'un verre de jus par jour, et pas tous les jours. Une grande majorité des participants ne connaît donc pas cette recommandation.

Concernant les apports nutritionnels des jus de fruits, $23 \%$ des participants pensent à tort que boire un verre de jus de fruit fournit les mêmes apports nutritionnels que de manger un fruit. Néanmoins, il est assez largement connu par les participants que la consommation de jus de fruit augmente les risques de maladies cardiovasculaires, de diabète et d'obésité ( $89 \%$ des participants). Enfin, $27 \%$ des participants pensent à tort que l'eau n'est pas la seule boisson recommandée.

\section{Des niveaux de connaissance nutritionnelle hétérogènes}

Depuis 2016, la réglementation européenne a rendu obligatoire les informations nutritionnelles sur la plupart des produits alimentaires. Les consommateurs sont donc régulièrement confrontés à ces informations sur les étiquettes des produits. Nous avons souhaité évaluer comment les consommateurs guadeloupéens perçoivent ces informations. Ainsi, plusieurs questions portaient sur la connaissance de termes de nutrition et la lecture d'étiquettes nutritionnelles.

La Fig. 6 présente les résultats des questions posées autour des connaissances en nutrition. Il apparaît que le terme «lipide » est peu compris par les participants : seulement $45 \%$ des individus ont su déchiffrer la quantité correcte de lipides sur l'étiquette nutritionnelle qui leur était présentée. Les notions de valeur énergétique et de sucre sont mieux maîtrisées par les participants, avec respectivement $70 \%$ et $68 \%$ de réponses correctes. Ce résultat peut s'expliquer par le fait que la quantité de lipides indiquée sur les étiquettes nutritionnelles est généralement appelée "matières grasses », contrairement à la valeur énergétique et à la quantité de sucre qui sont indiquées par ces mêmes termes.

Concernant la connaissance des termes «valeur énergétique » et «glucides », la Fig. 6 montre que respectivement $75 \%$ et $74 \%$ des participants maîtrisent leurs définitions. Cependant, la définition de l'allégation nutritionnelle «allégé en sucre » n'est pas connue par $87 \%$ des individus. Enfin, la quasi-totalité des participants ( $97 \%$ ) ne sait pas que les jus de fruits du commerce ne contiennent pas de sucres ajoutés.

\section{Étude des croyances alimentaires}

Pour identifier ce qui peut influencer les choix des consommateurs, nous nous sommes intéressées aux croyances alimentaires de la population guadeloupéenne. Les croyances alimentaires correspondent à ce que l'individu attend du produit et à l'idée qu'il s'en fait : par rapport à son origine, sa qualité, son goût, ses apports pour la santé, etc. Les croyances sont donc subjectives, hétérogènes et propres à chaque individu. 


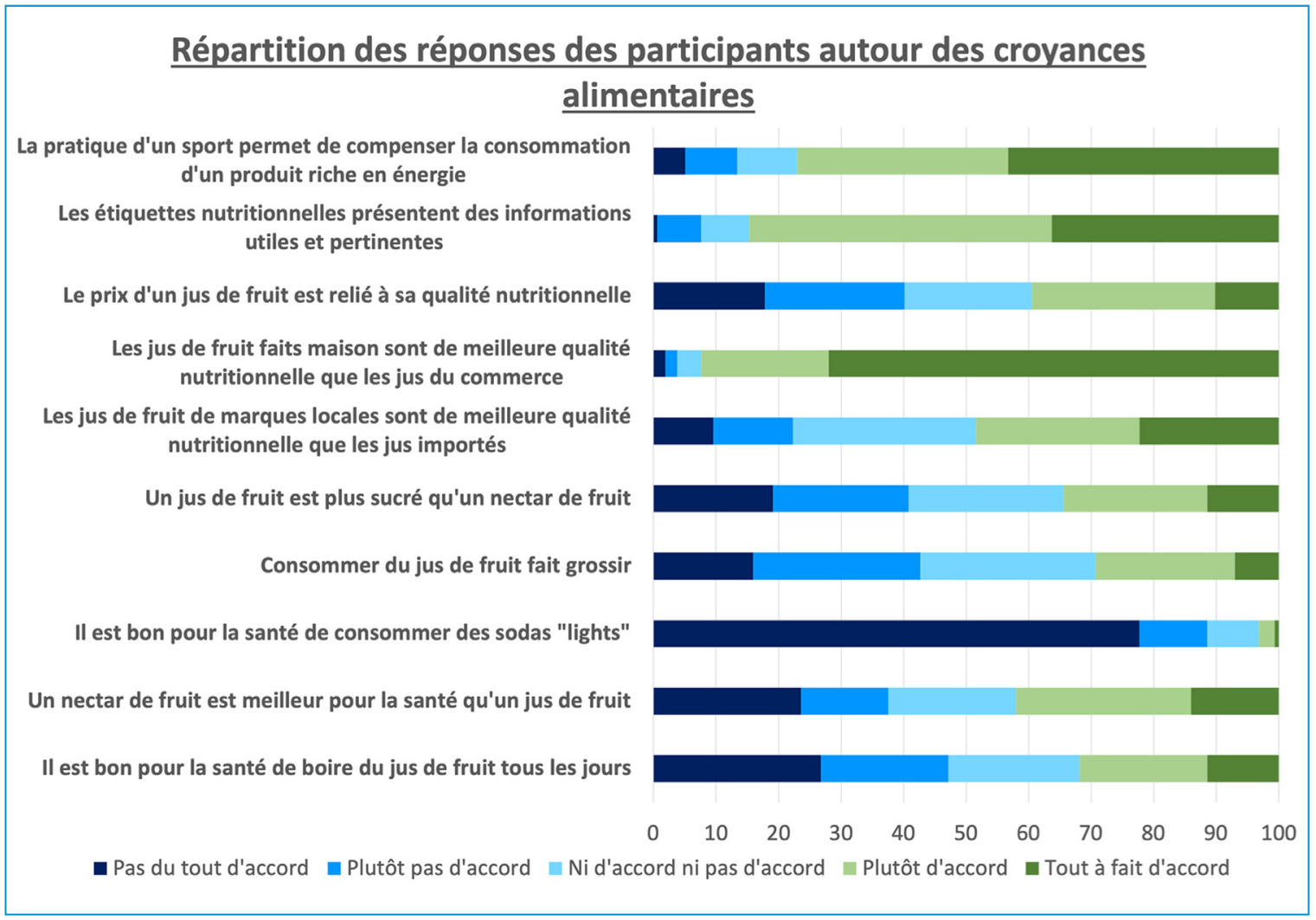

Figure 7. Répartition des réponses données par les participants concernant des affirmations relatives aux croyances alimentaires. $n=159$.

La Fig. 7 présente les résultats des questions autour des croyances alimentaires. Pour chaque affirmation posée, les individus ont été amenés à cocher leur degré d'accord selon une échelle en 5 points : Pas du tout d'accord - Plutôt pas d'accord - Ni d'accord ni pas d'accord - Plutôt d'accord - Tout à fait d'accord.

Il ressort que :

- $86 \%$ des participants voient dans les étiquettes nutritionnelles des informations utiles et pertinentes ;

- $93 \%$ des individus estiment que les jus de fruits maison sont de meilleure qualité nutritionnelle que ceux du commerce ;

- $89 \%$ des participants pensent que les sodas « light » ne sont pas bons pour la santé.

\section{Étude des habitudes alimentaires}

Une dernière partie du questionnaire était dédiée à l'étude des habitudes alimentaires de la population guadeloupéenne.

Les Fig. 8 et 9 présentent la fréquence de consommation de boissons à base de fruits des participants ainsi que les quantités consommées par prise. On peut noter que les $2 / 3$ des participants consomment des boissons à base de fruits au minimum plusieurs fois par semaine. Il apparaît en outre que seulement $7 \%$ des participants consomme la quantité maximale à consommer par jour recommandée par le PNNS, soit $10 \mathrm{cL}$ par jour et pas tous les jours.

\section{Déterminants des consentements à payer}

Dans cette partie, nous nous intéressons aux déterminants des consentements à payer. Il s'agit alors d'expliquer chaque consentement à payer révélé par les consommateurs en fonction de la teneur en sucres et de l'information dont il a disposé lors son évaluation. De cette façon il est possible d'estimer l'impact de l'information et du niveau de sucres sur les consentements à payer. Chaque participant ayant révélé 9 prix ( 3 nectars dans 3 situations informationnelles), nous disposons de 1451 observations de consentements à payer. Nous estimons cinq spécifications prenant en compte différentes variables explicatives dont les résultats sont présentés dans le Tableau $3^{5}$, chaque spécification a été estimée par la méthode des moindres carrés généralisés à effets aléatoires afin de prendre en considération l'hétérogénéité des préférences individuelles. Les résultats des régressions confirment les premières analyses menées à l'aide des tests non paramétriques.

La première spécification simple (colonne [1] dans le Tableau 3) estime les effets de la teneur en sucre et de la situation informationnelle sur la valorisation des consommateurs (exprimée par les consentements à payer). Ainsi, il apparaît une valorisation accrue du nectar le plus sucré, les consentements à payer des nectars à teneur en sucre moyenne (Med) et faible (Low) étant significativement plus

\footnotetext{
${ }^{5}$ Les résultats détaillés des estimations sont disponibles sur demande auprès des auteurs.
} 


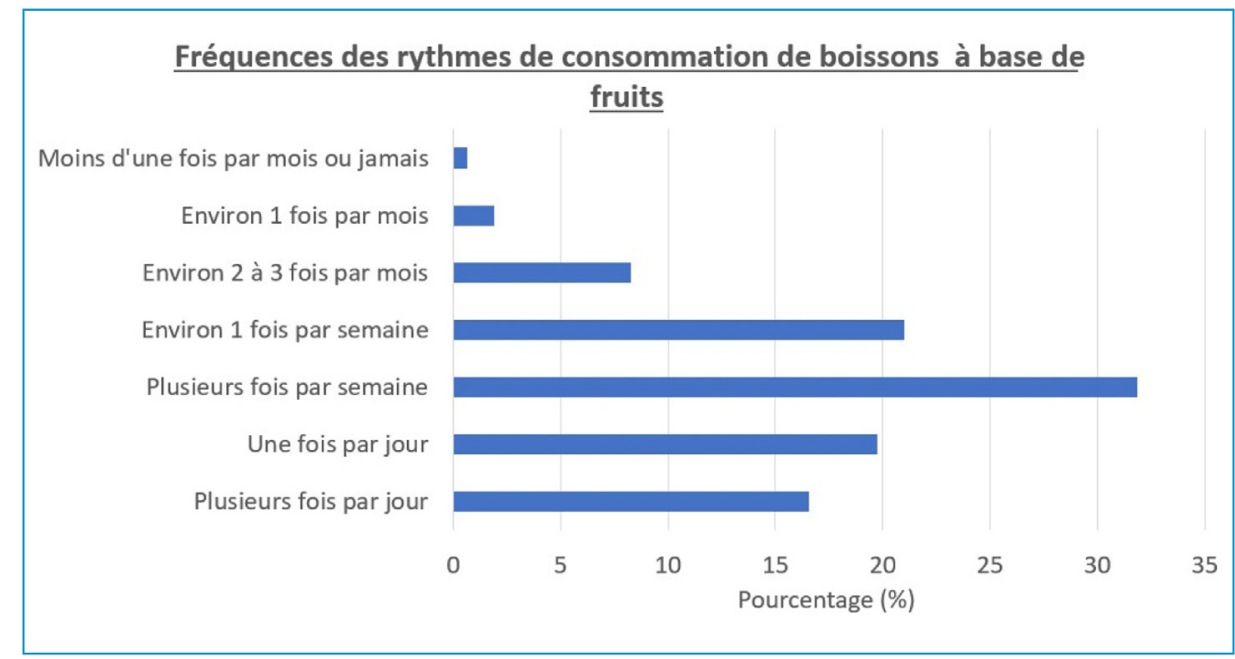

Figure 8. Fréquences des rythmes de consommation de boissons à base de fruits. $n=159$.

Fréquences des quantités consommées de boissons à base de fruit

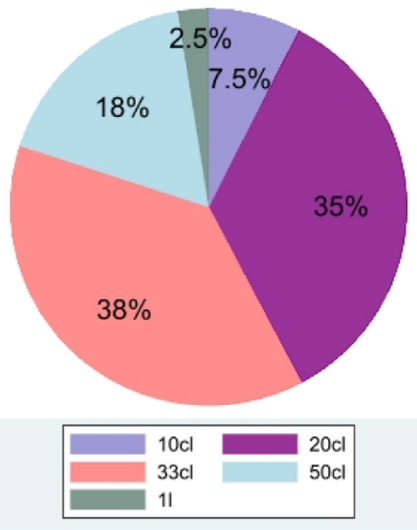

Figure 9. Fréquence des quantités de boissons à base de fruits ingérées par prise. $n=159$.

faible. Il apparaît également que les consentements à payer sont faiblement plus élevés lorsqu'ils ont été évalués en détenant la totalité de l'information (Full : étiquette et dégustation).

La deuxième spécification affine ces analyses en insérant les effets croisés entre la teneur en sucre et les situations informationnelles. En effet, l'effet de la teneur en sucre pouvant être différent selon la condition d'évaluation il semble important d'isoler ces effets. Cette spécification nous permet de confirmer l'effet de la teneur en sucre sur la valorisation des consommateurs. En effet, lors de la situation à l'aveugle les nectars à faibles teneurs en sucre sont significativement moins valorisés que celui à teneur en sucre élevée. Cependant, cette valorisation accrue du nectar le plus sucré est mise à mal lorsque les consommateurs révèlent leurs consentements à payer en ne disposant que des étiquettes (colonne [2] Tableau 3). Ainsi, non seulement le nectar le plus sucré est moins valorisé lors de l'estimation avec la seule étiquette mais il apparaît que les nectars aux teneurs moyenne et faible sont significativement plus valorisés lors de cette condition d'information. Enfin, il ne ressort pas d'effets croisés significatifs lors de la situation en évaluation complète. Cela implique donc que le fait de disposer de l'information complète ne modifie pas les arbitrages effectués en situation de dégustation à l'aveugle. Ainsi, dès lors que l'évaluation intègre la dimension sensorielle, l'information nutritionnelle ne permet pas compenser la valorisation moindre des nectars les moins sucrés.

La prise en compte des caractéristiques individuelles dans la valorisation des produits nous a semblé importante. Pour cette raison dans les trois spécifications suivantes nous avons introduit des variables individuelles.

Nous avons souhaité estimer si le niveau de connaissance des recommandations du PNSS avaient un impact sur la valorisation des nectars (colonne [3] Tableau 3). Pour cela nous avons créé un indice à partir des six questions présentées dans la section précédente. Il ressort de ces estimations que le niveau de connaissance du PNNS n'impacte pas la valorisation des consommateurs.

Par la suite, nous nous sommes concentrées sur le niveau de connaissances nutritionnelles afin de déterminer l'impact de celui-ci sur les niveaux de consentements à payer (colonne [4] Tableau 3). Là encore un indice à partir des questions décrites dans la section précédente (voir Fig. 6) et il ressort que le niveau de connaissance en nutrition n'impacte pas les consentements à payer des consommateurs.

Dans la dernière spécification présentée dans le Tableau 3, nous avons introduit les variables individuelles caractérisant nos participants, notamment le niveau de revenu, l'âge, le sexe et leurs fréquences de consommation de boissons à base de fruits. Aucune de ces variables n'a semblé montré d'impact significatif (au seuil de $5 \%$ ) sur la valorisation des nectars. Généralement, peu de variables sociodémographiques apparaissent comme significatives dans ce type d'études avec données expérimentales. Plus généralement, dans les études (quelles soit déclaratives, incitées ou issues de données de marché) s'intéressant aux préférences des consommateurs pour les allégations nutritionnelles ou santé, il n'y a pas de consensus sur l'effet des caractéristiques sociodémographiques. Le sexe, l'âge et 
Tableau 3 Signes des effets significatifs au seuil de $1 \%$ sur les consentements à payer. Taille de l'échantillon : 159 consommateurs, 1431 observations.

\begin{tabular}{|c|c|c|c|c|c|}
\hline Variables & $\begin{array}{l}(1) \\
\text { simple }\end{array}$ & $\begin{array}{l}(2) \\
\text { croisée }\end{array}$ & $\begin{array}{l}\text { (3) } \\
\text { recommandations }\end{array}$ & $\begin{array}{l}(4) \\
\text { nutrition }\end{array}$ & $\begin{array}{l}\text { (5) } \\
\text { Caractéristiques } \\
\text { individuelles }\end{array}$ \\
\hline High & ref & ref & ref & ref & ref \\
\hline Med & - & - & - & - & - \\
\hline Low & - & - & - & - & - \\
\hline Blind & ref & ref & ref & ref & ref \\
\hline Label & ns & - & - & - & - \\
\hline Full & + & ns & ns & ns & ns \\
\hline Med*Label & & + & + & + & + \\
\hline Med*Full & & ns & ns & ns & ns \\
\hline Low*Label & & + & + & + & + \\
\hline Low*Full & & ns & ns & ns & ns \\
\hline $\begin{array}{l}\text { Connaissances des } \\
\text { recommandations }\end{array}$ & & & ns & & \\
\hline Connaissances en nutrition & & & & ns & \\
\hline Homme & & & & & ref \\
\hline Femme & & & & & ns \\
\hline Moins de 452 euros & & & & & ref \\
\hline Entre 451 et 1200 euros & & & & & ns \\
\hline Entre 1201 et 2300 euros & & & & & ns \\
\hline Entre 2301 et 3800 euros & & & & & ns \\
\hline Entre 3801 et 4500 euros & & & & & ns \\
\hline Plus de 4500 euros & & & & & ns \\
\hline Moins de 30 ans & & & & & ref \\
\hline $30-39$ ans & & & & & ns \\
\hline $40-49$ ans & & & & & ns \\
\hline $50-59$ ans & & & & & ns \\
\hline Plus de 60 ans & & & & & ns \\
\hline $\begin{array}{l}\text { Consommateur fréquent ( } 1 \text { fois par } \\
\text { jour ou plus) }\end{array}$ & & & & & ref \\
\hline $\begin{array}{l}\text { Consommateur régulier (une ou } \\
\text { plusieurs fois par semaine) }\end{array}$ & & & & & ns \\
\hline $\begin{array}{l}\text { Consommateur occasionnel ( } 3 \text { fois } \\
\text { par mois ou moins) }\end{array}$ & & & & & ns \\
\hline Constant & + & + & + & + & + \\
\hline
\end{tabular}

Ref : catégorie de référence ; ns : non significatif.

le niveau d'éducation sont les variables les plus susceptibles d'avoir un impact significatif sur les préférences pour les allégations dans la littérature, cependant de nombreuses autres montrent que ces variables n'ont pas d'effet sur les intentions d'achats ou les préférences pour ces allégations nutritionnelles ou santé [20].

\section{Conclusion et discussion}

Dans cet article, nous nous sommes attachées à évaluer l'acceptabilité des consommateurs guadeloupéens pour des boissons sucrées à teneur en sucre plus faibles. En prenant en considération plusieurs dimensions des préférences, nous apportons des éclairages sur les arbitrages des consommateurs vis-à-vis des caractéristiques nutritionnelles et sensorielles. Nous montrons notamment que dès lors que les consommateurs intègrent la dimension sensorielle, la majorité d'entre eux marquent une préférence significative pour le produit le plus sucré. Néanmoins, la probabilité de préférer les produits les moins sucrés s'affirme au regard des seules informations nutritionnelles (présentation de la table nutritionnelle). On peut en déduire que les informations contenues dans les étiquettes nutritionnelles sont globalement bien interprétées par les consommateurs. Cela est en effet confirmé par les résultats obtenus sur l'analyse des connaissances élémentaires des participants sur les informations nutritionnelles (cf. voir la section Analyse descriptive des connaissances et croyances, réponses au questionnaire). Pourtant, lors de la révélation des préférences en ne disposant que de l'information donnée par l'étiquette (incluant la table nutritionnelle), on constate une hétérogénéité des préférences se traduisant par une distribution uniforme du nectar préféré entre les trois teneurs en sucres (cf. Fig. 3). 
Cela invite à s'interroger de manière plus approfondie sur les connaissances nécessaires à l'interprétation des informations nutritionnelles relativement à leur régime alimentaire global. S'il est à la portée des consommateurs de lire les étiquettes nutritionnelles, qu'en est-il de leur capacité à situer l'information dans un ordre de grandeur ? En somme que représentent en valeur absolue $10 \mathrm{~g}$ de sucres pour $100 \mathrm{~mL}$ ? Les consommateurs sont-ils capables d'apprécier s'il s'agit d'une teneur élevée ou non et d'appréhender les effets délétères sur la santé d'un tel taux lors de prises régulières?

Si comme le suggèrent [13], le déséquilibre alimentaire de la population d'Outre-mer pourrait provenir des quantités consommées, alors il convient d'approfondir notre analyse au regard de ces habitudes. Lorsqu'on s'intéresse aux habitudes de consommation déclarées par les participants, 68 \% d'entre eux déclarent consommer des boissons à base de fruit plusieurs fois par semaine (dont plus de la moitié consomment 1 ou plusieurs fois par jour). Il est notable que $50 \%$ des moins de 30 ans de notre échantillon déclarent consommer une boisson à base de fruits au moins une fois par jour. De plus lorsqu'on s'intéresse aux quantités consommées par prise, on constate également que les quantités ingérées sont largement au-dessus des recommandations du PNNS. En effet, presque $38 \%$ de notre échantillon déclarent consommer l'équivalent d'une canette de $33 \mathrm{cL}$ par prise de boisson à base de fruits et 17,61 \% déclare consommer $50 \mathrm{cL}$ par prise, ce qui, au regard de la teneur en sucres de ces boissons, est considérable en termes d'apports en sucres. Il est à noter que la consommation de boissons sucrées ne se limite pas qu'aux boissons à base de fruit, la consommation de sodas et autres boissons énergisantes peut, elle aussi, être une contributrice importante dans les apports en sucres.

Si l'idée d'une qualité nutritionnelle plus favorable ne provoque pas de rejet systématique, ce constat suggère plusieurs pistes d'approfondissements. Tout d'abord, il conviendrait d'analyser les préférences des consommateurs en intégrant une typologie plus fine des habitudes de consommations alimentaires comme variable individuelle explicative. En effet, la prise en compte des variables individuelles n'étant pas probante dans nos analyses, il pourrait être pertinent d'intégrer un indicateur de consommation combinant les fréquences d'achat et les quantités consommées par prise dans les analyses des préférences et des arbitrages afin de déterminer dans quelle mesure ces habitudes sont explicatives des préférences. Les résultats de [17] qui montrent la prédominance des habitudes et de la dimension hédonique dans les choix alimentaires, laissent à penser que cette piste est pertinente. De plus, la prise en compte des spécificités individuelles par une analyse par segment de consommateurs pourrait être une piste intéressante pour approfondir l'importance des caractéristiques individuelles. Enfin, la prise en compte, d'une part, du décalage des fréquences de consommation et des quantités consommées avec les recommandations du PNNS, et d'autre part, la méconnaissance de ces recommandations (seuls $12 \%$ de notre échantillon connaissait la quantité maximale de jus de fruit recommandée par jour, à savoir $10 \mathrm{cL}$ par jour et pas tous les jours) laisse à penser que les campagnes informationnelles doivent être adaptées. Or comme le soulignent [21], l'éducation à l'alimentation doit être multidimensionnelle et doit notamment intégrer le plaisir alimentaire en promouvant la sensorialité alimentaire (ce qui peut aboutir à une diminution des tailles de portions) et l'aide à la gestion de la relation à l'alimentation. Il nous semblerait donc pertinent d'intégrer ces résultats dans la mise en place de mesures d'éducation à l'alimentation.

\section{Remerciements}

Cette étude a été réalisée dans le cadre du projet NuTWind [ANR-16-CE21-0009] financé par l'Agence Nationale de la Recherche, France. Les auteurs remercient l'unité expérimentale $\mathrm{PEYI}$ et plus particulièrement Katy Begarin et Jean-Louis Diman pour leur appui logistique et administratif.

\section{Déclaration de liens d'intérêts}

Les auteurs déclarent ne pas avoir de liens d'intérêts.

\section{Références}

[1] Méjean C, et al., editors. Alimentation et nutrition dans les départements et régions d'Outre-mer. IRD Éditio. Marseille: IRD; 2020 [208 p. Expertise collective, bilingue françaisanglais].

[2] Maire B, Lioret S, Gartner A, Delpeuch F. Transition nutritionnelle et maladies chroniques non transmissibles liées à l'alimentation dans les pays en développement. Cah Etude Rech Francoph 2002;12(1):45-55.

[3] ORSAG. Comportements alimentaires et perceptions de l'alimentation en Guadeloupe en 2010, Vol. 2010; 2010.

[4] Colombet Z, Allès B, Perignon M, Landais E, MartinPrevel $\mathrm{Y}$, Amiot $\mathrm{MJ}$, et al. Caribbean nutrition transition: what can we learn from dietary patterns in the French West Indies? Eur J Nutr [Internet] 2021;60(2):1111-24, http: / /dx.doi.org/10.1007/s00394-020-02317-x.

[5] Colombet Z, Perignon M, Salanave B, Landais E, Martin-Prevel $Y$, Allès $B$, et al. Socioeconomic inequalities in metabolic syndrome in the French West Indies. BMC public health 2019;19(1):1-11.

[6] Allais $O$, Bertail P, Nichèle $V$. The effects of a fat tax on French households' purchases: a nutritional approach. Am J Agric Econ 2010;92(1):228-45.

[7] Réquillart V, Bonnet C. Que faut-il attendre des taxes nutrtionnelles? INRA Sci Soc 2014;9(111):4.

[8] Boizot-Szantaï C, Etilé F. Taxer les boissons sucrées pour lutter contre l'obésité ? Le point de vue de l'économie. Obésite 2011;6(4):218-25.

[9] Crosetto P, Lacroix A, Muller L, Ruffieux B. Modification des achats alimentaires en réponse à cinq logos nutritionnels. Cah Nutr Diet [Internet] 2017;52(3):129-33, http://dx.doi.org/10.1016/j.cnd.2017.04.002.

[10] Crosetto P, Lacroix A, Muller L, Ruffieux B. Nutritional and economic impact of five alternative front-of-pack nutritional labels: experimental evidence. Eur Rev Agric Econ 2020;47(2):785-818.

[11] Muller L, Prevost $M$. What cognitive sciences have to say about the impacts of nutritional labelling formats. J Econ Psychol [Internet] 2016;55:17-29, http://dx.doi.org/10.1016/j.joep.2016.01.005.

[12] Reif X. Conditions de vie en 2018 : en Guadeloupe, deux personnes sur cinq sont en privation matérielle et sociale. INSEE Anal 2020;46:4. 
[13] Soler LG, Eymard Duvernay S, Rollet P, Ducrot A, Terrieux $P$, Amélie K, et al. L'offre de boissons, de produits laitiers frais et de biscuits est-elle vraiment plus sucrée aux Antilles qu'en France hexagonale? Cah Nutr Diet 2021;56(6), http://dx.doi.org/10.1016/j.cnd.2021.08.001.

[14] Combris P, Ruffieux B. La révélation expérimentale des préférences des consommateurs. INRA Sciences Sociales; 2005.

[15] Combris P, Bazoche P, Giraud-Héraud E, Issanchou S. Food choices: what do we learn from combining sensory and economic experiments? Food Qual Prefer 2009;20(8):550-7, http://dx.doi.org/10.1016/j.foodqual.2009.05.003.

[16] Canavari M, Drichoutis AC, Lusk JL, Nayga RM. How to run an experimental auction: a review of recent advances. Eur Rev Agric Econ [Internet] 2019;46(5):862-922 [Available from: https://mpra.ub.uni-muenchen.de/89715/].

[17] Merdji M, Certenais E, Mairesse G, Kerhoas N, Mourot J, Dourmad JY. Perception of messages associated with three sample menus in a company restaurant: healthy, low carbon footprint and traceable. Cah Nutr Diet [Internet] 2019;54(4):230-9, http://dx.doi.org/10.1016/j.cnd.2019.04.001.

[18] Bernard JC, Liu Y. Are beliefs stronger than taste? A field experiment on organic and local apples. Food Qual Prefer [Internet] 2017;61:55-62, http://dx.doi.org/10.1016/j.foodqual.2017.05.005.

[19] Becker GM, DeGroot MH, Marschak J, Becker GM, DeGroot MH, Marschak J. Measuring utility by a single-response sequential method. Behav Sci 1964;9(3):226-32.

[20] Steinhauser J, Hamm U. Consumer and product-specific characteristics influencing the effect of nutrition, health and risk reduction claims on preferences and purchase behavior - A systematic review. Appetite [Internet] 2018;127:303-23, http://dx.doi.org/10.1016/j.appet.2018.05.012.

[21] Bertin E. For a three-dimensional approach to nutrition education. Cah Nutr Diet 2020;55(3):119-26. 